\title{
High frequency vibration of inverter-fed PMSM and its reduction
}

\author{
Tao Wang ${ }^{1, a}$, Haixiang $\mathrm{CaO}^{1, \mathrm{~b},}$ Zhanlu Yang ${ }^{1, \mathrm{c}}$ and Shanming Wang ${ }^{1, \mathrm{~d}}$ \\ ${ }^{1}$ State Key Lab of Control and Simulation of Power Systems and Generation Equipment, Dept. of \\ Electrical Engineering, Tsinghua University, Beijing 100084, China

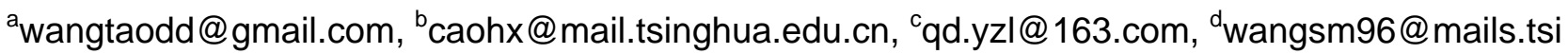 \\ nghua.edu.cn
}

Keywords: PMSM; high-frequency vibration; electromagnetic exciting force; frequency.

\begin{abstract}
Permanent magnet synchronous motors (PMSM) generate high-frequency electromagnetic vibration and noise when powered by inverters, and the cause is high-frequency electromagnetic exciting force (EMEF). In this paper, the frequencies and orders of EMEF are analyzed. The expression of EMEF acted on the stator inner surface is derived and the frequencies and orders of main components of vibration are listed. The high-frequency vibration experiments are conducted on a surface mounted 8-pole and 12-slot PMSM. The high-frequency vibrations near switch frequency and its multiples are main components of vibrations of motors when powered by inverters, and experiments are conducted to study the influence of switch frequency on the vibration acceleration. The impacts of periodic frequency PWM and random frequency PWM on the vibration acceleration and peak value of its frequency spectrum are studied by experiments.
\end{abstract}

\section{Introduction}

The vibration of motors is one of the most important performance indicators. The noise generated by vibration will pollute our working and living environment. A severe vibration can even destruct the machine structure. There are higher requirements for vibration in special applications. While the application of inverter improves the dynamic performance of the motor, it causes high frequency vibration and noise. Many researchers have done a lot of work about the vibration and noise of motors. The frequency of noise of inverter-fed induction machine is calculated[1,2]. The influence of inverters on the vibration of axial flux PM motors is studied by finite element method(FEM) and experiments. The impacts of random PWM and harmonic injection is studied[4-6]. The effect of pulse frequency modulation(PFM) in reducing noise of induction motor is studied[7].

When powered by inverters, the stator current has a lot of time harmonics which increases the complexity of air gap flux. The air gap flux determines the magnitude and frequency of electromagnetic exciting force (EMEF) acted on the inner surface of stator. In this paper, the flux distribution is derived by magnetic circuit method firstly. Then, the expression of radial of EMEF is obtained by Maxwell's stress tensor. The frequency, order and sources of the main EMEF are listed. The high-frequency vibration experiments are conducted on a surface mounted 8-pole and 12-slot PMSM. The high-frequency vibrations near switch frequency and its multiples are main components of vibrations of motors when powered by inverters, and experiments are conducted to study the influence of switch frequency on the vibration acceleration. The impacts of periodic frequency PWM and random frequency PWM on the vibration acceleration and peak value of its frequency spectrum are compared by experiments.

\section{Calculation of EMEF in PMSM}

The vibration of stator of PMSM is mainly determined by radial EMEF acted on the inner surface of the stator. Neglecting the effect of magnetostriction and assuming $\mu_{F e} \gg \mu_{0}$, the EMEF acted on the inner surface can be expressed as[8]: 


$$
F_{n}=\frac{1}{2 \mu_{o}} B_{0 n}^{2}
$$

where $B_{0 n}$ is the radial air gap flux density in the inner surface of stator. So the air gap flux distribution need to be obtained before calculating the EMEF.

Neglecting the core magnetic reluctance, the air gap flux distribution can be expressed as

$$
b(\theta, t)=f(\theta, t) \cdot \lambda(\theta, t)
$$

where $\theta$ is the mechanical angle, $f(\theta, t)$ is the air gap magneto-motive force(MMF) and $\lambda(\theta, t)$ is the air gap permeance.

In a surface mounted PMSM (SPMSM), the air gap permeance does not change with time. It can be approximately expressed as

$$
\lambda(\theta)=\Lambda_{0}+\sum \lambda_{l}
$$

where $\Lambda_{0}$ is the constant part and $\lambda_{l}$ is the space periodic part caused by slots. $\lambda_{l}$ can be expressed as

$$
\lambda_{l}=\Lambda_{l} \cos (l Z \theta)=\frac{\mu_{0}\left(k_{\delta}-1\right)}{k_{\delta} \delta}\left|\frac{\sin \frac{\left(k_{\delta}-1\right) l \pi}{k_{\delta}}}{\frac{\left(k_{\delta}-1\right) l \pi}{k_{\delta}}}\right| \cos (l Z \theta)
$$

where $k_{\delta}$ is Carter's coefficient, $\delta$ is the length of air gap and Z is the number of slots.

The MMF of inverter-fed PMSM includes the 5 following parts as equation (5): (a) the fundamental MMF caused by stator fundamental current and PM; (b) the space harmonic MMF caused by stator fundamental current; (c) the equivalent harmonic MMF of PM; (d) the space fundamental MMF caused by harmonic current; (e) the space harmonic MMF caused by harmonic current.

$$
\begin{aligned}
& f_{0}(\theta, t)=F_{0} \cos \left(p \theta-\omega_{0} t-\varphi_{0}\right) \\
& \sum f_{v}(\theta, t)=\sum F_{v} \cos \left(v \theta \pm \omega_{0} t-\varphi_{v}\right) \\
& \sum f_{\mu}(\theta, t)=\sum F_{\mu} \cos \left(\mu p \theta-\mu \omega_{0} t-\varphi_{\mu}\right) \\
& \sum f_{k}(\theta, t)=\sum F_{k} \cos \left(p \theta-k \omega_{0} t-\varphi_{k}\right) \\
& \sum f_{k v}(\theta, t)=\sum F_{k v} \cos \left(v \theta \pm k \omega_{0} t-\varphi_{k v}\right)
\end{aligned}
$$

where $F$ is the magnitude of MMF, $p$ is the pole pair number, $\omega_{0}$ is the fundamental angle frequency, $v$ is the order of stator space harmonic MMF, $\mu$ is the order of rotator space harmonic MMF and $k$ is the order of harmonic current. When a motor is supplied with sinusoidal current, there is no MMF caused by harmonic current. When a motor is powered by an inverter, the output harmonic voltage can generate harmonic current of the same frequency. The output voltage of a three-phase inverter can be calculated by double Fourier integral and expressed as

$$
\begin{aligned}
u_{A B} & =\frac{\sqrt{3}}{2} M E_{d} \cos \left(\omega_{0} t-\varphi-\frac{\pi}{3}\right) \\
& -\frac{4 E_{d}}{\pi} \sum_{k_{1}=1,2 \ldots k_{2}= \pm 1, \pm 3 .}^{\infty} \sum_{k_{1}}^{+\infty} \frac{J_{k_{2}}\left(\frac{k_{1} M \pi}{2}\right)}{k_{1}} \sin \left(\frac{k_{1}+k_{2}}{2} \pi\right) \sin \left(\frac{k_{2}}{3} \pi\right) \cos \left[\left(k_{1} N+k_{2}\right) \omega_{0} t-k_{2} \varphi+\frac{k_{2}}{3} \pi-\frac{k_{2}}{2} \pi\right]
\end{aligned}
$$

where $\mathrm{M}$ is the modulation ratio, $E_{d}$ is dc-bus voltage, $J_{n}$ is a Bessel function, $N$ is carrier wave ratio. According to equation(6), the frequency of output harmonic current of inverter is

$f_{k}=k_{1} f_{s} \pm k_{2} f_{0}$

where $f_{s}$ is the switch frequency, $f_{0}$ is the fundamental frequency, $k_{1}$ and $k_{2}$ are positive integers of different parity. In a three-phase inverter, $k_{2}$ is not the multiples of three. 
Substituting equation (3)-(5) into equation(2), the air gap flux of inverter-fed PMSM is expressed as

$$
b(\theta, t)=f(\theta, t) \cdot \lambda(\theta, t)=\left(\begin{array}{l}
F_{0} \cos \left(p \theta-\omega_{0} t-\varphi_{0}\right)+ \\
\sum F_{v} \cos \left(v p \theta \pm \omega_{0} t-\varphi_{v}\right)+ \\
\sum F_{\mu} \cos \left(\mu p \theta-\mu \omega_{0} t-\varphi_{\mu}\right)+ \\
\sum F_{k} \cos \left(p \theta-k \omega_{0} t-\varphi_{k}\right)+ \\
\sum F_{k v} \cos \left(v \theta \pm k \omega_{0} t-\varphi_{k v}\right)
\end{array}\right) \times\left(\Lambda_{0}+\sum \Lambda_{l} \cos (l Z \theta)\right)
$$

Combining equation(1) with equation(8), the EMEF of an inverter-fed SPMSM is obtained. Because the magnitude of harmonic MMF caused by harmonic current is small, it is neglected in calculation. When calculating the permeance, only the term $l=1$ is considered. As the vibration response is inversely proportional to $4^{\text {th }}$ power of order of EMEF, only the EMEF with large magnitude and low order is considered to analyze the vibration of motors. After the simplification, the main EMEFs, their sources, orders and frequencies are listed in Table 1.

Table 1 Order and frequency of radial EMEF of inverter-fed motors

\begin{tabular}{|c|c|c|c|c|}
\hline Current & Source 1 & Source 2 & Order & Frequency \\
\hline \multirow{9}{*}{$\begin{array}{l}\text { fundamental } \\
\text { current }\end{array}$} & Fundamental magnetic field & Fundamental magnetic field & $2 p$ & $2 f_{0}$ \\
\hline & Fundamental magnetic field & Stator $v_{\text {th }}$ harmonic magnetic field & $(v \pm 1) p$ & $2 f_{0}$ \\
\hline & Fundamental magnetic field & Rotator $\mu_{t h}$ harmonic magnetic field & $(\mu \pm 1) p$ & $(\mu \pm 1) f_{0}$ \\
\hline & Fundamental magnetic field & $\begin{array}{l}\text { Fundamental MMF and harmonic } \\
\text { nermeance }\end{array}$ & $(2 p-Z)$ & $2 f_{0}$ \\
\hline & Fundamental magnetic field & Stator $v_{t h}$ MMF and harmonic & $(v \pm 1) p-Z$ & $2 f_{0}$ \\
\hline & Fundamental magnetic field & Rotator $\mu_{t h}$ MMF and harmonic & $(\mu \pm 1) p-Z$ & $(\mu \pm 1) f_{0}$ \\
\hline & Stator $v_{1 t h}$ harmonic magnetic field & Stator $v_{2 t h}$ harmonic magnetic field & $\left(v_{1}-v_{2}\right) p$ & $2 f_{0}$ \\
\hline & Rotator $\mu_{1 t h}$ harmonic magnetic & Rotator $\mu_{2 t h}$ harmonic magnetic & $\left(\mu_{1}-\mu_{2}\right) p$ & $\left(\mu_{1}-\mu_{2}\right) f_{0}$ \\
\hline & Stator $v_{t h}$ harmonic magnetic field & Rotator $\mu_{\text {th }}$ harmonic magnetic & $(v-\mu) p$ & $(\mu \pm 1) f_{0}$ \\
\hline \multirow{4}{*}{$\begin{array}{l}\text { Harmonic } \\
\text { current }\end{array}$} & Fundamental magnetic field & Fundamental magnetic field of $k_{t h}$ & 0 or $2 p$ & $(k \pm 1) f_{0}$ \\
\hline & Fundamental magnetic field of $k_{1 t h}$ & Fundamental magnetic field of $k_{2 t h}$ & 0 or $2 p$ & $\left(k_{1}-k_{2}\right) f_{0}$ \\
\hline & Fundamental magnetic field of $k_{t h}$ & Stator $v_{t h}$ harmonic magnetic field & $(v \pm 1) p$ & $(k \pm 1) f_{0}$ \\
\hline & Fundamental magnetic field of $k_{t h}$ & Rotator $\mu_{t h}$ harmonic magnetic field & $(\mu \pm 1) p$ & $(k \pm \mu) f_{0}$ \\
\hline
\end{tabular}

According to Table 1, when powered by sinusoidal current, the order of vibration is associated with pole-pair number, order of space harmonic magnetic field and slot number, the frequency is even-multiples of fundamental frequency. When powered by inverters, the inverters cause $0_{t h}$ and $2 p_{\text {th }}$ high frequency vibration and the frequency $f_{n}$ is

$$
f_{n}=f_{k} \pm f_{0}=k_{1} f_{s} \pm k_{3} f_{0}
$$

where $k_{1}$ and $k_{3}$ are integers of same parity.

\section{Experiments of high frequency vibration of PMSM}

To verify the calculation of frequency of EMEF, the vibration of an 8-pole and 12-slot PMSM is measured when it is operating with no load at different speeds. Setting the $f_{s}$ as $10 \mathrm{kHz}$, the fundamental frequency as $60 \mathrm{~Hz}$ and the rotating speed as $900 \mathrm{rpm}$, the current waveform is shown as Fig. 1 and it contains a lot of high frequency harmonics. The frequency spectrum of current near $10 \mathrm{kHz}$ is shown as Fig. 2. The magnitude of harmonic current is normalized to fundamental current. 
According to the frequency spectrum, the frequencies of harmonic current are correspondent with equation(7).

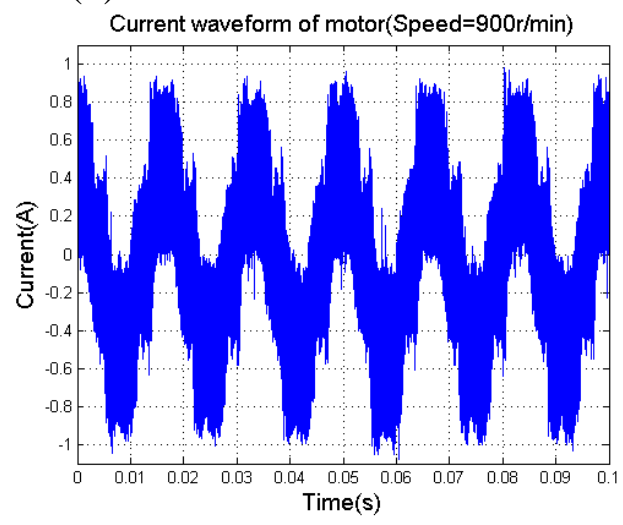

Fig. 1 Current waveform of motor at 900rpm

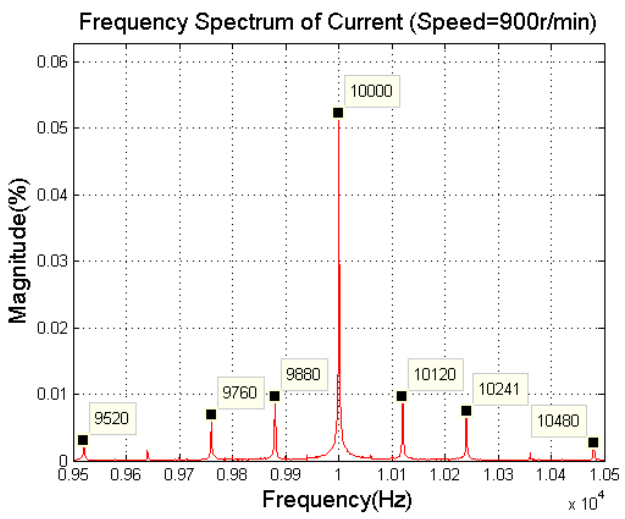

Fig. 2 Frequency spectrum of current at 900rpm

The outer surface vibration of motor is measured by accelerometer and the waveform is shown as Fig.3. The fundamental frequency of vibration is $120 \mathrm{~Hz}$ which is 2 times of fundamental frequency of current. The frequency spectrum of vibration is shown as Fig. 4. The high frequency vibrations near $f_{s}$ and its multiples are main components. The frequency spectrum near $10 \mathrm{kHz}$ is shown as Fig. 5 and the frequency of each harmonic is correspondent with equation(9). The vibrations of the motor from 0 to 900rpm are measured and the frequency spectrum is shown as Fig. 6. In Fig. 6, the medial axis is the switch frequency $10 \mathrm{kHz}$. With the increase of speed, the frequency spectrum separate further and the magnitudes of vibration increase. At different speed, the frequency of each high frequency vibration harmonic is correspondent with equation(9).

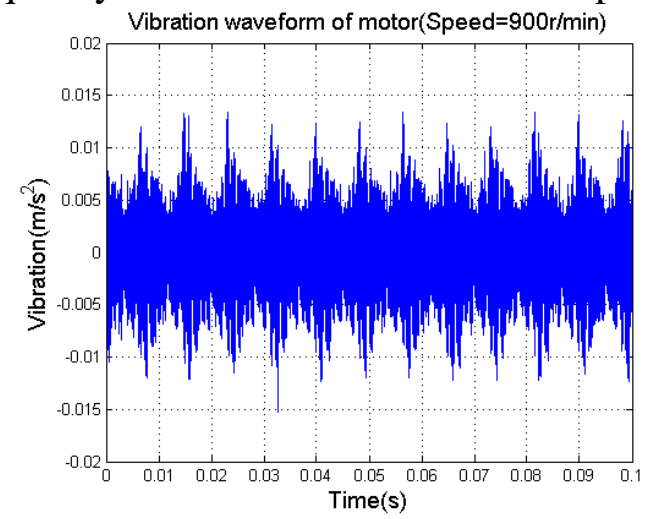

Fig. 3 Acceleration waveform of motor

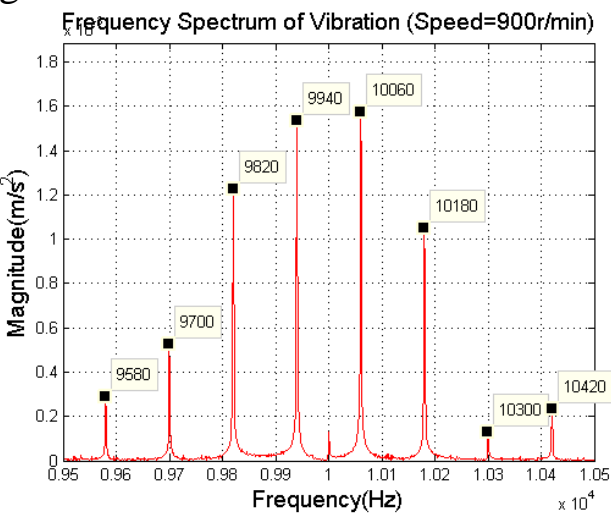

Fig. 5 Frequency spectrum of vibration near $10 \mathrm{kHz}$

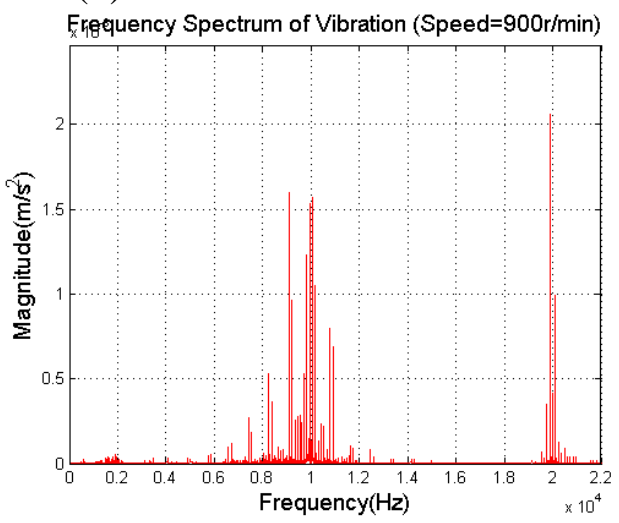

Fig. 4 Frequency spectrum of acceleration

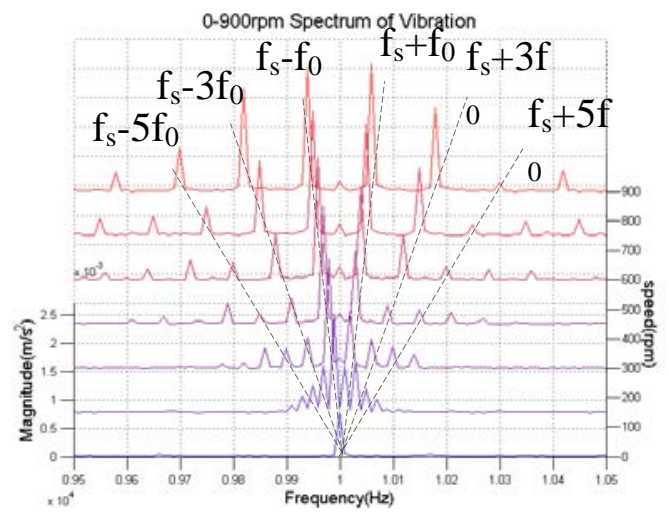

Fig. 6 Frequency spectrum at different speeds 


\section{Influence of switch frequency on high frequency vibration}

According to Fig. 4, the switch frequency has great influence on the high frequency vibration of motors when powered by inverters. Equation(6) shows that the harmonic voltage magnitude of $k_{1} f_{s}+k_{2} f_{0}$ is a Bessel function of $k_{1}, k_{2}$ and has no relevance to $f_{s}, f_{0}$. However, the impedance of stator windings $R+j \omega L$ is approximately determined by the reactance $j \omega L$ which is proportional to the frequency. Therefore, with the increase of $f_{s}$, the impedance of $k_{1} f_{s}+k_{2} f_{0}$ will increase and the corresponding harmonic current will decrease. The harmonic current is approximately inversely proportional to its frequency. Fig. 7 is an experimental result of the relationship between harmonic current magnitudes of $f_{s}+2 f_{0}$ and $f_{s}$ and the result proves the theoretical analysis. The radial EMEF which causes high frequency vibration is the interaction between fundamental magnetic field and space fundamental magnetic field caused by high frequency harmonic current. Therefore, with the increase of $f_{s}$, the magnitude of high frequency harmonic current will decrease and so will the EMEF. However, the analytical calculation or simulation of the relationship between high frequency response and switch is not available because of the complex mechanical structures of motors. Experiments are conducted to measure the influence of the $f_{s}$ on the vibration acceleration $a_{r m s}$.
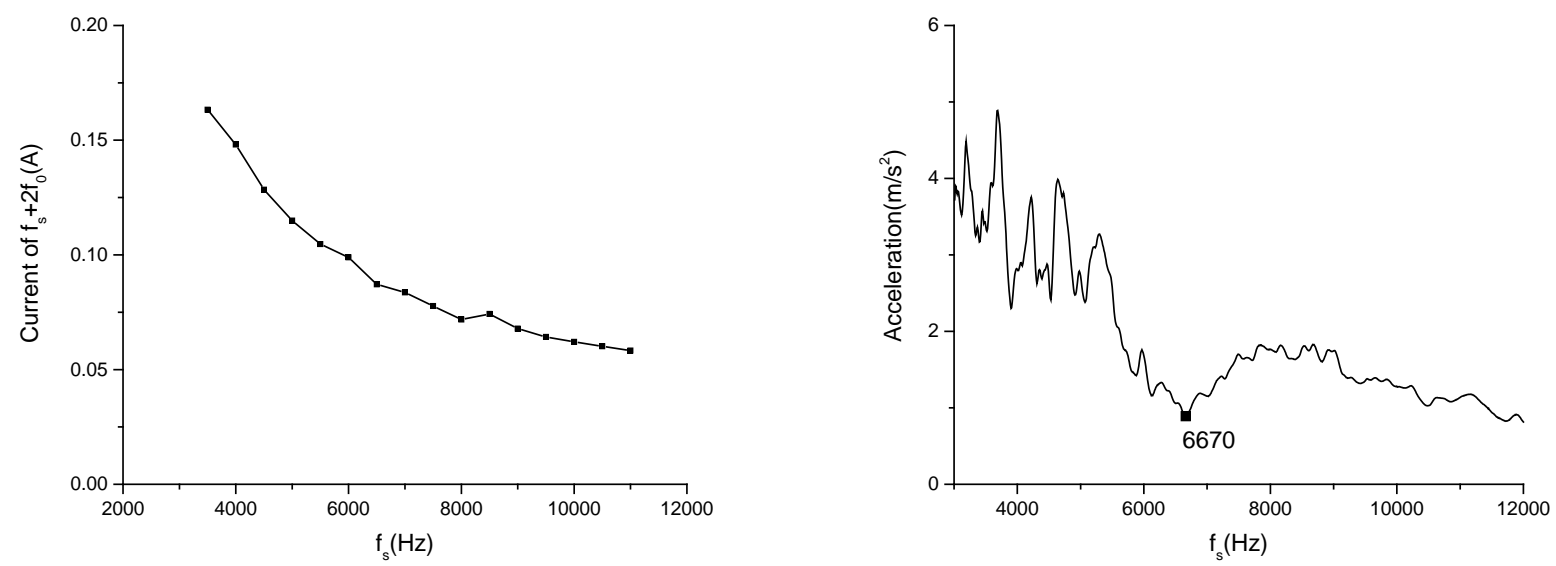

Fig. 7 Relationship between harmonic current and $f_{s}$ Fig. 8 Relationship between vibration and $f_{s}$

The outer surface vibration acceleration is measured while the switch changes from $3 \mathrm{kHz}$ to $12 \mathrm{kHz}$. The result is shown in Fig. 8. The acceleration shows a downtrend because of the decease of the EMEF as the $f_{s}$ increases. Due to the mechanical characteristics of motor, the acceleration shows an obvious fluctuation in the range of $3 \mathrm{kHz}-5 \mathrm{kHz}$. At the point of $6670 \mathrm{~Hz}$, the acceleration is the lowest point in the range of $3 \mathrm{kHz}-11.5 \mathrm{kHz}$. Increasing the frequency will reduce the high frequency EMEF so as to reduce the vibration, but it will also increase the power loss of the semiconductors. While there exists an upper limit of $f_{s}$, Fig. 8 has an important reference value to choose an optimal $f_{s}$ which makes the vibration least.

\section{Effect of variable switch frequency PWM on high frequency vibration}

Besides the $a_{r m s}$, the peak value of the frequency spectrum of acceleration $a_{\text {peak }}$ is also an important indicator of vibration performance. When the $f_{s}$ is fixed, the vibration power focuses on the frequencies of $k_{1} f_{s} \pm k_{3} f_{0}\left(k_{1}, k_{3}\right.$ are integers of same parity). The variable switch frequency PWM can spread the vibration power within a frequency band and reduce the peak value. Periodic switch frequency PWM (PSFPWM) and random switch frequency PWM (RSFPWM) are two common variable switch frequency techniques.

PSFPWM adds a relatively smaller periodic component to the fixed $f_{s}$. The new switch frequency is expressed as 


$$
f_{s}=f_{s 0}+R(t) \Delta f
$$

where $f_{s 0}$ is a fixed central frequency, $R(t)$ is a periodic function with a output range [-1,1] and $\Delta f$ is the bandwidth of variable frequency. During one period, the frequency changes from $f_{s 0}-\Delta f$ to $f_{s 0}+\Delta f$. Setting $f_{s 0}=6670 \mathrm{~Hz}, \Delta f=500 \mathrm{~Hz}, R(t)$ as a triangular function, experiments are conducted to compare the vibration under this condition and fixed frequency $6670 \mathrm{~Hz}$. The experimental results are shown as Fig. 9 and Fig. 10. When the $f_{s}$ is a fixed value $6670 \mathrm{~Hz}$, the $a_{\text {peak }}$ is $0.77 \mathrm{~m} / \mathrm{s}^{2}$ at the frequency $6850 \mathrm{~Hz}$ while the $a_{r m s}$ is $1.07 \mathrm{~m} / \mathrm{s}^{2}$. When PSFPWM is applied, the $a_{\text {peak }}$ drops significantly to $0.14 \mathrm{~m} / \mathrm{s}^{2}$ while the $a_{r m s}$ rises to $1.17 \mathrm{~m} / \mathrm{s}^{2}$. The spectrum lines are approximately distributed uniformly within the range $5880 \mathrm{~Hz}-7460 \mathrm{~Hz}$. The result shows that the PSFPWM can reduce the $a_{\text {peak }}$ but will increase the $a_{r m s}$.

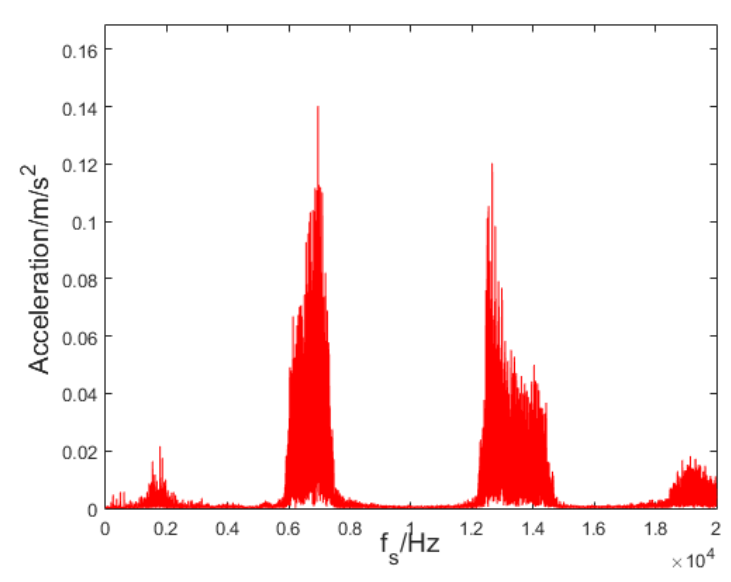

Fig. 9 Frequency spectrum of PSFPWM

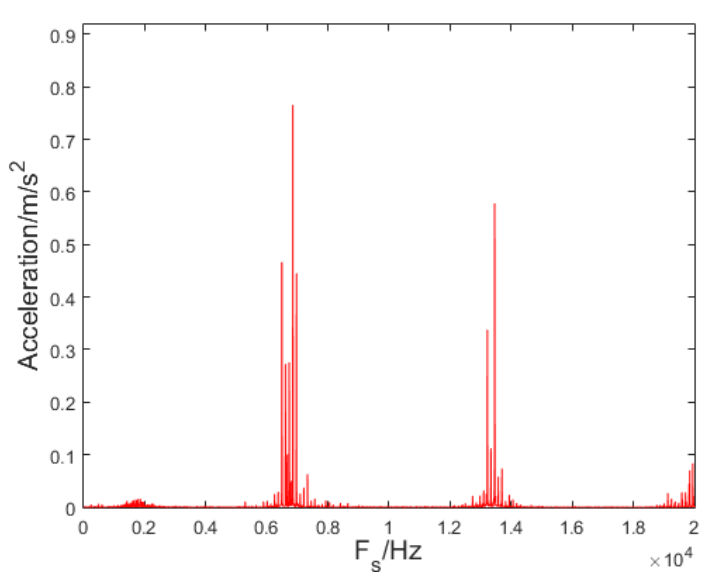

Fig. 10 Frequency spectrum of $6670 \mathrm{~Hz}$

Similar to PSFPWM, the frequency of RSFPWM is expressed as

$$
f_{s}=f_{s 0}+\operatorname{rand}() \Delta f
$$

where $f_{s 0}$ is a fixed central frequency, rand () is a random function with a output range $[-1,1]$ and $\Delta f$ is the bandwidth of variable frequency. Then the $f_{s}$ is a random value within the range $\left[f_{s 0}-\Delta f, f_{s 0}+\Delta f\right]$. Setting $f_{s 0}=6670 \mathrm{~Hz}, \Delta f=500 \mathrm{~Hz}$, rand () as a uniform random function with 20 points, the vibration of motor with the RSFPWM technique applied is measured. The frequency spectrum of acceleration is shown as Fig. 11. Compared to Fig. 10, the $a_{r m s}$ increases slightly from $1.07 \mathrm{~m} / \mathrm{s}^{2}$ to $1.09 \mathrm{~m} / \mathrm{s}^{2}$, but the $a_{\text {peak }}$ decreases from $0.77 \mathrm{~m} / \mathrm{s}^{2}$ to $0.14 \mathrm{~m} / \mathrm{s}^{2}$. The spectrum lines are distributed in the whole frequency domain.

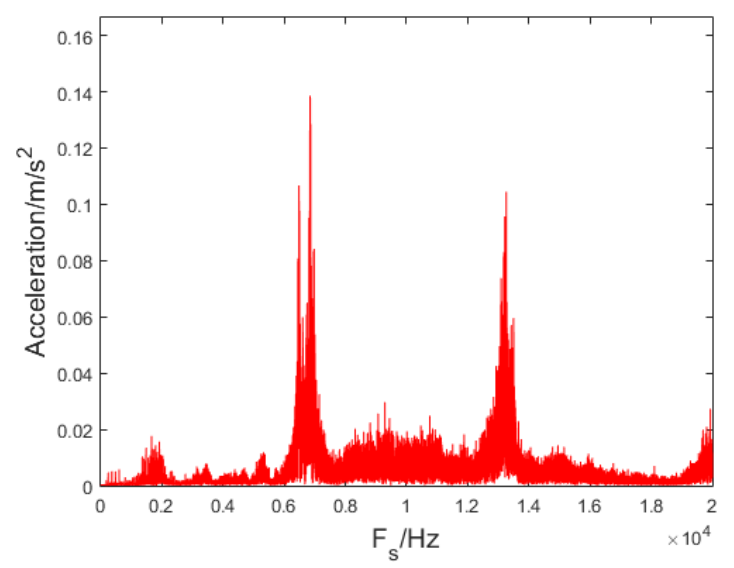

Fig. 11 Frequency spectrum of RSFPWM

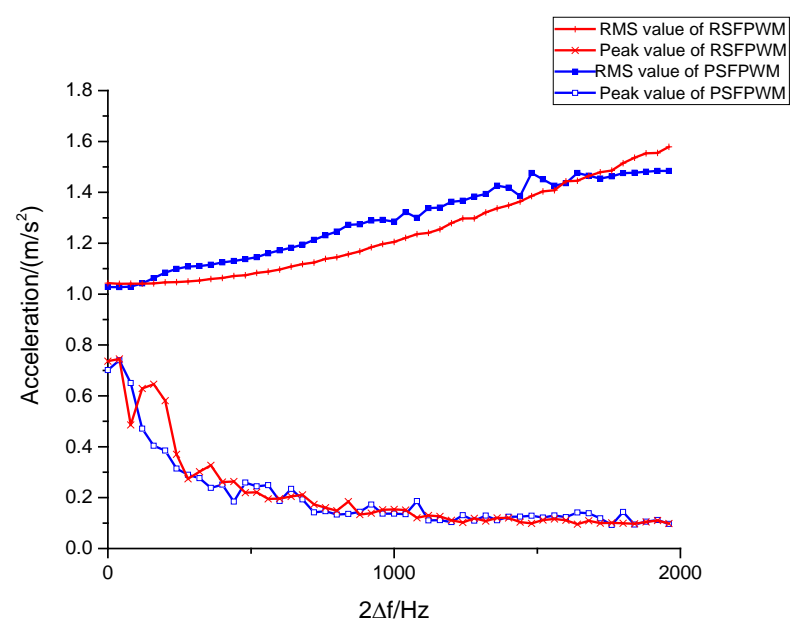

Fig. 12 Relationship between $\Delta f$ and $a_{r m s}, a_{\text {peak }}$ 
In both variable switch frequency PWM techniques, the bandwidth of frequency $\Delta f$ is a very important factor. Experiments are conducted to study the impacts of $\Delta f$ on the $a_{r m s}$ and $a_{\text {peak }}$ in both PSFPWM and RSFPWM. The result is shown in Fig. 12. As the $\Delta f$ increases, the two curves of peak value of frequency spectrum of acceleration show the same trend and they both decrease significantly and then tend to be constants. The two curves of $a_{r m s}$ both rise gradually as the $\Delta f$ increases but have a little difference in magnitude. Within the range of $0 \mathrm{~Hz}-900 \mathrm{~Hz}$, the $a_{r m s}$ of RSFPWM is smaller than PSFPWM.

According to Fig. 8, the frequency $6670 \mathrm{~Hz}$ is the locally optimal frequency of the certain 8-pole 12- slot PMSM which makes the vibration least. And according to Fig. 12, the RFPWM technique with parameter $\Delta f=500 \mathrm{~Hz}$ can reduce the $a_{\text {peak }}$ and only increases the $a_{r m s}$ slightly. Therefore it is an effective method to reduce the vibration of inverter-fed PMSM.

\section{Summary}

The air gap flux density of an inverter-fed SPMSM is derived and the radial EMEF acted on the inner surface of stator is obtained. When powered by inverters, the high frequency vibration of motors is mainly caused by the interaction between fundamental magnetic field and space fundamental magnetic field caused by high frequency harmonic current. The frequency is $f_{n}=k_{1} f_{s} \pm k_{3} f_{o}\left(k_{1}, k_{3}\right.$ are integers of same parity) and the calculation is verified by experiments. The high-frequency vibrations near $f_{s}$ and its multiples are main components of vibrations of motors when powered by inverters. The $f_{s}$ has great influence on the high frequency vibration of motors. The acceleration shows a downtrend generally and there exists a locally optimal frequency $6670 \mathrm{~Hz}$ which makes the vibration least due to the mechanical characteristics of motor. Both PSFPWM and RSFPWM can effectively reduce the $a_{\text {peak }}$ and increase the $a_{r m s}$ slightly. The larger the bandwidth of variable frequency is, the smaller the $a_{\text {peak }}$ will be and the larger the $a_{r m s}$ will be. According to the relationship between $f_{s}$ and $a_{r m s}$, an optimal $f_{s}$ which makes the $a_{r m s}$ least can be chosen out. Then according to the relationship between $\Delta f$ and $a_{\text {peak }}, a_{r m s}$, a suitable $\Delta f$ can be chosen out. The RFPWM technique with optimal parameters $f_{s}$ and $\Delta f$ will be an effective method to reduce the vibration of inverter fed PMSM. 


\section{References}

[1] Lo W C, Chan C C, Zhu Z Q,et al. Acoustic noise radiated by PWM-controllel induction machine drives[J]. Industrial Electronics, IEEE Transactions on, 2000, 47(4):880-889.

[2] Belmans R J M, Verdyck D, Geysen W,et al. Electro-mechanical analysis of the audible noise of an inverter-fed squirrel-cage induction motor[J]. Industry Applications, IEEE Transactions on, 1991, 27(3):539-544.

[3] Tang Renyuan, Song Zhihuan, Yu Shenbo, et al. Study on source of vibration and acoustic noise of permanent magnet machines by inverter[J]. Electric Machines and Control,2010,(03): 12-17.

[4] Ruiz-Pe X, Rez J, Ruiz-Gonza X,et al. A new RPWM technique with harmonics injection and frequency randomized modulation[C]. Electrical Machines (ICEM), 2010 XIX International Conference on, 2010:1-6.

[5] Borisov K, Calvert T E, Kleppe J A,et al. Experimental investigation of a naval propulsion drive model with the PWM-based attenuation of the acoustic and electromagnetic noise[J]. Industrial Electronics, IEEE Transactions on, 2006, 53(2):450-457.

[6] Lo W C, Chan C C, Zhu Z Q,et al. Acoustic noise radiated by PWM-controllel induction machine drives[J]. Industrial Electronics, IEEE Transactions on, 2000, 47(4):880-889.

[7] Ki-Seon K, Young-Gook J, Young-Cheol L. Shaping the Spectra of the Acoustic Noise Emitted by Three-Phase Inverter Drives based on the New Hybrid Random PWM Technique[C]. Power Electronics Specialists Conference, 2006. PESC '06. 37th IEEE, 2006:1-6.

[8] Ruiz-Gonzalez A, Meco-Gutierrez M J, Perez-Hidalgo F,et al. Reducing Acoustic Noise Radiated by Inverter-Fed Induction Motors Controlled by a New PWM Strategy[J]. Industrial Electronics, IEEE Transactions on, 2010, 57(1):228-236.

[9] He Guhuan. Analysis of electromagnetic vibration-and-noise of permanent-magnet direct current commutator motors[D]. Shanghai Jiao Tong University, 2012.

[10]Zhang Ran. Research of electromagnetic exciting force of surface-mounted permanent magnet motors and its reduction methods[D]. Shandong University, 2011.

[11]Pan Gaoqiang. Study on pwm harmonic analysis and harmonic suppression method for inverter[D]. Shenyang University of Technology, 2010.

[12]Chen Qiuming, Chen Yong. Study on Theory of Electro-Magnetic Vibration and Noise of a Permanent Magnet Synchronous Motor[J]. Small \& Special Electrical Machines, 2013,(08): 1-5. 